\title{
Using Academic Social Networks to Enhance the Student Experience in Online Education
}

\author{
Tiffani S. Bateman \\ University of Phoenix, USA
}

\begin{abstract}
Online universities utilize academic social networks to build connections among students, faculty, and alumni through affinity groups. This study explored how students interact in academic social networks, who they collaborate with, why they use academic social networks, and how this influences their educational experience. This qualitative, interpretive, phenomenological study explored the lived experiences of six online higher education students reporting active participation in an academic social network. Three core themes emerged from data analysis: (a) acceptance and belonging; (b) self-validation; and (c) drawing from multiple perspectives describing how academic social networking communities are formed, why students are using them, and what this means to online higher education. The essence of academic social networking as it relates to self-actualization is discussed, with insights for educational leaders regarding the use of academic social networking and affinity groups in online higher education.
\end{abstract}

Keywords: Social networking; online learning; online education; phenomenology

Bateman, T. (2021). Using academic social networks to enhance the student experience in online education. Online Learning, 25(4), 295-323. DOI: 10.24059/olj.v25i4.2532 
Despite the sharp increase in enrollment in online learning over the past few decades, retention continues to be one of the greatest challenges educators face in distance education (Oregon, et al., 2018). Finding methods to reduce student attrition rates is critical for online higher education universities. The primary factors to consider in understanding the high dropout rates in distance education include identifying which students are dropping out and what factors influence this decision (Radovan, 2019). One of the reasons that students report dropping out of online education programs is the lack of social presence (Ali \& Kohun, 2007; Ivonkova \& Stick, 2007). Learning and the acquisition of knowledge is a social process. Social presence is the degree of connectedness individuals feel when communicating through any given medium across space or time (Rogers, et al, (2009). It is therefore critical to understand how students communicate and interact socially in online education, and the implications of this for student persistence.

Despite lower completion rates than traditional brick and mortar universities, student demand for online education programs is increasing (Oregon, et al., 2019). Distance learning provides students an opportunity to earn their degrees when an on-ground institution is either not an option or a desire. Different communication mediums provide for different perceptual experiences of social presence in distance learning. Academic social networks may hold the key to providing students with a perception of social connectedness, which may result in increased student satisfaction as well as increased retention rates for online higher education institutions. Therefore, institutions embrace the use of social networks, hoping to enhance the educational experience for online students (Kabilan, et al., 2010; Valdez, et al., 2020).

Students report feeling more engaged in online learning when utilizing various technologies (Educause Center for Applied Research, 2010). Communication mediums such as social networks attract millions of users and allow for a unique social experience that defies space and time. Understanding student experiences with, and attitudes toward, academic social networking may help educational leaders realize the potential of this tool in building social presence in the online university (Rajagopal, et al., 2012). Empirical research is limited in providing institutions with insights regarding the benefits or drawbacks of such a social medium (Liu, et al., 2010; Rajagopal, et al., 2012), despite finding that students with a stronger sense of social presence and community have a higher satisfaction with their learning experience (Akyol et al, 2008) which in turn may have a positive impact on student retention. This research aims to gain a better understanding of how students are using these academic social networks and what impact this has on their online learning experience.

\section{Purpose of the Study and Research Questions}

The purpose of this study is to understand the online student experience in academic social networks. By examining both posting and non-posting behavior in academic social networks, educators can better understand new forms of communication and how information is exchanged. How students use these networks may impact their perception of the education they receive as well as their sense of community within a given institution. Such experiences may directly influence the likelihood of students persisting in their education through the attainment of a degree. A responsive interviewing model was implemented to gain in-depth insight into each participant's experience. One overarching and exploratory research question was used to guide the study: What meaning does the experience of academic social networking hold for online higher education students? 
Three broad sub-questions were utilized to draw out the data needed to cover the context of the phenomena of the study (Smith et al., 2009).

1. What is the student experience when using an academic social network?

2. Who or what do students observe in the academic social network?

3. What benefits or drawbacks have students experienced from using an academic social network?

\section{Review of Relevant Literature}

Academic social networking is defined as a private educational community network that allows members to collaborate, communicate, and exchange educational information, ideas, and views where members cannot otherwise meet face-to-face (Glezou, et al., 2010). Research regarding general social networking in the context of education was utilized to build a theoretical framework due to the gap in the literature on academic social networking. The framework of the study includes the socio-ecological theory, socio-constructive learning theory, and community of inquiry.

The study is framed in a socio-ecological perspective which embraces the notion of a reciprocal relationship between the student and the learning environment where the student shapes his or her intrapersonal environment while simultaneously transforming the self (Altback, et al., 2005). Student learning occurs over a wide range of contexts in informal and formal settings situated in socio-cultural theory whereas learning is "located in contexts and relationships rather than merely in the minds of individuals" (Greenhow, et al., 2009, p. 248). Epistemological assumptions surrounding socio-ecological theory include learning as a derivative of participation in communal activities tied to social experiences (Greenhow et al., 2009).

Academic social networks provide a unique virtual space where communication and collaboration unfold. As such, digital technologies such as academic social networks have the power to influence the learner and the instructor but also impact and redefine the administrative functions of institutions (Saykili, 2019). In this study, the socio-ecological lens provides a layer to explore and analyze the relationship between the student and the community.

Socio-constructivist learning theory provides a second lens to frame this research. This lens recognizes that an academic social network serves as a mediator by which students can collaborate and share ideas and form relationships. Socio-constructive learning theory is consistent with such an idea as knowledge is a product of the environment and context where learning takes place (Hofstetter \& Schneuwly, 2009). The epistemological assumptions guiding socio-constructive learning theory include the idea that group interactions, experiences, and individual interactions advance knowledge through collaborations unique to the medium or environment where they occur.

The community of inquiry (CoI) theory has been used over the past decade in hundreds of studies regarding online education (Arbaugh et al., 2008) and provides the third lens for this study. Garrison et al. (2010) surveyed over 200 students across 14 educational institutions and found students perceived social presence to be a significant factor influencing cognitive presence and learner outcomes. Ke (2010) reported a positive correlation between social presence and cognitive presence in the online learning environment. Student self-perceived sense of community is also positively correlated to learning satisfaction (Ke, 2010). Social presence plays 
a vital role in both teaching presence and cognitive presence which directly affects student satisfaction with learning.

The philosophical principles of phenomenology include the idea that inquiry into an experience through the perception of an individuals' experience will unveil a deeper truth (Smith et al., 2009). Various perspectives allow for multi-layered perceptions of the academic social networking phenomenon. By layering academic social networking within the context of a socioecological framework, socio-constructive learning theory, and a community of inquiry framework, an understanding of the students' experience of academic social networking is and what this phenomenon means within the context of online learning.

\section{History of Social Networking}

A national study from Pew Internet \& American Life Project reported that college students frequently utilize the internet with $73 \%$ of users reporting active social network use (Jones, 2002). Understanding how college students use social network sites for social interaction, communication, connections, and relationships provides valuable information concerning the future of social networking and academic social networking. A sample of 258 undergraduate students from a western university in the United States completed a survey administered to determine the demographic characteristics and social networking involvement (Bahk et al., 2010). Seventy-nine percent of respondents reported utilizing social networking sites approximately five hours a week.

Ilkyu and Chonggun (2014) conducted a study utilizing the social networking site Twitter for educational purposes in two classes at Yeungnam University. The experiment included students and professors utilizing Twitter for the following educational purposes: (a) students can ask questions and professor can answer and post for all the class to see, and (b) professor can suggest a non-graded discussion topic and students can post their opinions. For the two experimental classes using Twitter, findings indicated that students with more followers and followings received higher grades and students who participated received higher grades than those who did not participate.

Eiodice and Gaffin (2008) investigated correlations between Facebook postings and student achievement in a study of undergraduate Zoology students. A high correlation between photos posted on the social network site and high academic achievement revealed students more engaged and networked with peers tended to be higher achievers academically (Eiodice \& Gaffin, 2008). Lower academic performance was also highly correlated with the number of Facebook applications downloaded, suggesting that applications without educational merit hold little value in academic social networks.

An online panel of 351 consumers participated in a study to identify what demographic characteristics and personality traits differed between posters and non-posters in social networking sites (Morrison \& McMillian, 2010). Individuals active in social networks reported lurking behaviors, or observing the interactions of other online users, more frequently than posting content, with women reporting more frequent use of social networking sites than men. Of those participants that do visit social networking sites, participants who were older reported less activity on social networks than students under 25 years of age (Morrison \& McMillian, 2010). Findings indicated individuals scoring higher on the extroversion scale were also more likely to be active in social networking, whether displaying posting or lurking behaviors (Morrison \& McMillian, 2010). 
Social network sites first gained popularity from colleges and universities for marketing purposes and universities began to experiment with how social network sites (SNSs) might be used as marketing tools (College Board and Art \& Science Group, 2009). A small number of universities have since created their own SNS, or academic social network, allowing students to blog, tag, and access various SNS functions within the university website (Kaya, 2010). The Academic Commons of The City University of New York (CUNY) is a SNS designed for students, alumni, and faculty to create networks and communicate ideas in an evolving social community (Kaya, 2010). CUNY registered members create profiles, post information, and join groups online to collaborate with colleagues and peers from the university.

Similar academic social networks hosted by universities include the University of Pennsylvania's College of Liberal and Professional Studies Open Learning Commons, Emory University's LearnLink, and the University of Phoenix's Phoenix Connect, among others. With these newly formed academic social networkers, researchers are now able to gather data concerning the user experience in academic SNSs. Such data may provide insight toward a new level of connectedness and engagement not previously explored in academia within the context of social networking.

\section{Educational Networking}

Educational networking refers to social networks used in educational environments or for educational purposes (Creative Commons Attribution Share-Alike 3.0, 2011), and over 600 educational networks exist around the globe. Some of these educational networks are academic social networks and others are places to explore technology, find educational groups, or find similar interest groups within larger social networks such as Facebook. Although so many educational networks exist, the idea is relatively new, with Vicki A. Davis first proposing the idea of using social networks for educational purposes in 2008 in an online debate on social networking technologies in education hosted by The Economist (Boyd, 2011).

Despite minimal research on academic social networks, researchers identify benefits to social networking as related to educational purposes. A study conducted by researchers at the University of Minnesota (2008) found that students using social networks for personal use are practicing the 21 st-century skills educators wish to develop. Ninety-four percent of students aged 16 to 18 reported using the internet with $77 \%$ having a profile on a social network site.

Arnold and Paulus (2010) conducted a qualitative research study examining student perceptions of social networking when implemented in a course using Ning®. Perspectives from the students and instructor as well as an outside observer provided data for this investigation. The site created in Arnold and Paulus's study enabled students to upload pictures to their profiles, which students reported made them feel more a part of a community than in courses in which this feature was not available (2010). Students also reported that the site made it easier to contact peers outside of class for such needs as missed notes, help on work, or study groups (Arnold \& Paulus, 2010).

\section{Formal and Informal Learning}

Informal online learning is the pursuit of knowledge, skill, or understanding occurring externally without the utilization of an imposed curricular objective or criteria which happens in daily life when accessing the Internet (Livingstone, 2001; Holland, 2019). The National Centre for Education Statistics (NCES) started collecting data on adults' informal learning in 2005, 
recognizing lifelong learning to be a critical factor in the growth and development of a global knowledge economy (National Centre for Educational Statistics, 2006; Rubenson \& Desjardin, 2009; Smith \& Smith, 2008). Findings from analysis of international data since this conception suggest "informal learning is particularly important to support disadvantaged adults who may face barriers in accessing more structured learning activities" (Heo \& Lee, 2013, p. 411).

Heo and Lee (2013) studied over 10,000 adults and found the preferred method for informal learning is through sharing and interpersonal communication. Data analysis revealed a significant difference between males' and females' value of informal learning. Women reported a higher level of enjoyment when participating in informal learning activities, such as informal learning on the internet, than men. Bahk, Sheil, Rohm, and Lin (2010) reported similar findings, with women reporting more frequent use of social networks than their male counterparts.

Chen and Bryer (2012) investigated the utilization of social media in formal and informal learning contexts. Formal learning makes up only $8 \%$ of learning for undergraduate students and $5 \%$ of learning during the graduate years (Banks et al., 2007). Learning happens everywhere and at any time, often because of interactions between peers, co-workers, and the environment. Social networks have the capacity to connect formal learning in the traditional classroom to informal learning in and connect students in meaningful ways (Chen \& Bryer, 2012). Understanding the processes which contribute to informal learning in social networks will enable educators to create rich and meaningful learning experiences (Heo \& Lee, 2013). Twenty-first century educators serve as guides, using their expertise to guide students through "coaching, mentoring, knowledge-sharing, and team teaching" (Saykili, 2019, p. 6).

\section{Social Networking Behavior}

Little documentation exists regarding lurking behavior, yet three-quarters of respondents' report lurking behavior as opposed to posting when visiting social networking sites, suggesting that lurkers seek out social networking sites although their presence in the site goes undetected (Morrison \& McMillian, 2011). Such implications raise questions as to what draws social networkers to such sites. Demographics such as household income, education, and ethnicity did not differ in the panel of consumers studied in either posters or lurkers. Lurking is the number one behavior reported in social networking (Edelmann, 2013). Edelmann (2013) challenges researchers to situate lurkers in a positive light, as valuable participants in academic social networks. Understanding why individuals lurk will provide valuable insight for online behavior in social networks.

Muller et al., (2009) suggest lurkers may be a hidden asset in online communication. Chen and Chang (2013) studied lurkers in small group online communities to better understand why these individuals visit the site and what their influence is on learning within the group. Lurkers are classified differently from study to study and not necessarily defined as individuals who have never posted (Lin \& Tsai, 2011). Chen and Chang (2013) analyzed 82 small groups of senior and junior high social networkers participating in a virtual science fair. Lurkers included infrequent posters, non-posters, and sporadic posters in the social network across the 82 groups. Findings suggest lurkers "are highly contributive to collaborative knowledge building" and a valid form of participation in online communities (Chen \& Chang, 2013, p. 22). Further findings suggest that, as opposed to a vicarious learner, lurkers engage in critical areas of discussion by opening complicated discussions that engage other, more frequent posters, suggesting that frequency of posting may not be the best indicator of learning in the online social network. 
In response to inquiries regarding lurking behavior in online learning, the E-Listening Project was initiated in 2011 to investigate how these behaviors may influence learning. Research is focused on exploring the multidimensional aspects involved in lurking behavior and suggests a need to examine the invisible behavior of online learners in greater detail (The EListening Project, 2011). The notion of lurking, which suggests a negative connotation related to lack of contribution, has been replaced in recent literature with the notion of online listening.

Cyberloafing is the act of using information technology tools in a work or school environment for personal reasons (O’Neill, et al, 2014). In an education context, cyberloafing includes the time students spend in academic social networks. Studies conducted on cyberfloating in an educational context focus largely on how social networks may cause negative behaviors such as procrastination (Gerow, et al, 2010) and cognitive absorption (Tanriverdi et al, 2018). "In general, related literature is not much focused on variation of cyberloafing behaviors at education environments" (Durak, 2020, p. 540). There is a gap in research regarding the student experience when using academic social networks.

\section{Methods}

The study employed a qualitative method to explore how online higher education students experience academic social networking. An interpretive phenomenological design focused on the lived experiences of the individuals in the context of the phenomenon of study (Merriam, 2009; Smith, Flowers, \& Larkin, 2009). The research is framed by the philosophical assumption that reality is socially constructed based on individual interpretations. The purpose of constructing new knowledge by combining unique ontological perspectives with consideration to the researcher's own ontological bias is consistent with a socio-constructive epistemology and phenomenological methodology (Merriam, 2009).

Specifically, an interpretive phenomenological analysis aims to focus on the details of a phenomenon and how those details are understood by individuals within a specific context (Smith, et al., 2009). Interviews serve as the data-gathering tool for the study. Data collected through interviews account for participant perceptions of lived experiences when using an academic social network and is open to interpretation based on participant ontology. The broader online population may not perceive the same experiences, which reduces the ability to generalize results to a larger population. This phenomenological study focuses on rich and detailed accounts of the experience derived from a small population, meant to explain a phenomenon as opposed to replicate or generalize the results (Neuman, 2006).

\section{Phenomenological Approach}

A phenomenological design was utilized in the study to investigate human experiences and derive knowledge from real world experiences (Merriam, 2009). A phenomenological research design is used to extrapolate common themes based on rich textural descriptions of the students' lived experiences to gain understanding about their use of academic social networks. The focus of this phenomenological study was to uncover the initial meaning behind the experience as it exists in the consciousness of the individual (Moustakas, 1994).

\section{Participants}


The study population consisted of online higher education students actively involved in an academic social network. Active enrollment includes all students who have completed a minimum of 24 consecutive credit hours and are currently enrolled in an online degree program. This population included students across various degree programs at various levels of degree completion. Participants came from international locations since the unique experience of participating in an academic social network leaves no constraints as to physical geography. The only geographic stipulation was that the university offer fully online degrees options without students having to be physically present on campus.

Students were emailed an online student survey (see Appendix A), a request for voluntary participation, and an informed consent form, which required an electronic signature. Students who returned an electronically signed informed consent form were considered when identifying the final study sample. Potential interviewees were provided a survey asking for demographic and degree i program information. Information regarding time spent in an academic social network (see Table 1) was also collected. The survey contained background information regarding what constitutes active involvement in an academic social network and asked students to elaborate on their own experiences.

\section{Table 1}

\begin{tabular}{llll}
\multicolumn{2}{l}{ Variation of Study Participants } \\
\hline Participant & $\begin{array}{l}\text { Time Spent in Academic } \\
\text { Social Network }\end{array}$ & $\begin{array}{l}\text { \# of Years in } \\
\text { Online Education }\end{array}$ & $\begin{array}{l}\text { Degree Program } \\
\text { (Bachelor's) }\end{array}$ \\
\hline 1 & $15 \mathrm{~min} /$ day & 4 & Business Management \\
2 & $2-3$ times/week & 5 & Information Technology \\
3 & $4-5$ times/week & 3 & Information Technology \\
4 & $60 \mathrm{~min} /$ week & 2 & Criminal Justice \\
5 & $15 \mathrm{~min} /$ week & 4 & Business Management \\
6 & $15 \mathrm{~min} /$ week & 3 & Health Administration \\
\hline
\end{tabular}

Students were chosen purposefully for participation based on the information provided in the survey. Information-rich cases were selected based on the level of activity participants reported utilizing an academic social network. The most important criterion for participant selection was identifying participants who provided a detailed account of their experiences using academic social networks.

The sample size for this study was a total of six participants $(n=6)$. Students who reported active involvement, both in posting and non-posting, or lurking, behavior were selected to participate in the study to gather multiple perspectives from different instances of the phenomenon. Students with a wide variety of backgrounds and characteristics were considered as is consistent with maximum variation sampling (Merriam, 2009).

\section{Instrumentation and Data Collection}

Incorporating the responsive interviewing model, interview questions were divided into main questions, probes, and follow-up questions throughout the interview to both clarify what was heard and gain information about gaps which came to light during the process. Students were asked to remember what they discussed with other individuals in these networks and how 
that made them feel about the learning experience. The interview guide contained the following broad interview questions: (1) What are your experiences when using an academic social network? (2) Who or what do you observe in the academic social network? (3) What benefits or drawbacks have you experienced from using an academic social network?

The interview guide was reviewed by a panel of three researchers in the field of social networking and online education prior to beginning the interviews and no changes were suggested. By listening to and exploring students' lived experiences, themes emerged wherein follow-up questions allowed the interviewer to expand upon interviewee experiences relevant to the context of the research. Omissions regarding the experience led to follow-up questions or prompts which provided additional clarity.

Audio recordings were used to capture the details of participants' responses and later transcribed. Each participant was given the opportunity to review the transcript of his/her interview to fortify validity and accuracy. Each interviewee was given the opportunity to add any additional information although none did.

\section{Data Analysis}

Each individual transcript was analyzed serially beginning with student 1 (S1) and ending with student 6 (S6). Semantic language and content exploration involved a detailed analysis of each transcription. Initial noting began with the conceptual comments noted in the journal alongside the ideas bracketed off during the actual interview. Initial noting was captured in the left-hand column of the transcripts using different features such as italics, highlights, and underlining to organize which noting strategies mapped to specific text.

Several strategies aided in the listing and preliminary grouping of the text during the initial noting. Descriptive comments captured content in key words or phrases used by the participants. Conceptual comments included more overarching details of the participant's experience of the phenomenon. The analytic process of horizontalization was utilized to identify each statement, concept, and response, hence building a textual description of the phenomenon for each participant. The hermeneutic cycle is evident in this step as the whole transcript was reorganized and chunked during this process of reduction and elimination. Overlapping statements and redundancies were eliminated. Each horizon or invariant constitute was reviewed to ensure it could be labelled and was relevant to understanding the phenomena. Horizons not meeting these requirements were eliminated.

During data analysis, several similar horizons were combined and renamed as a broad understanding of the meaning behind these combined emergent themes. Horizons were clustered to develop superordinate themes within each case. In some instances, subsumption was utilized whereas an emergent theme was reorganized to become a superordinate theme. A listing of emergent themes for each participant allowed the researcher to demonstrate how clusters formed around superordinate themes.

These eight superordinate themes were analyzed across all cases, consistent with the hermeneutic cycle. Superordinate themes shared higher order concepts in some instances and were relabeled accordingly. Emergent themes such as "support network of peers with similar interests from different backgrounds," "seeking guidance," and "career networking" were combined along with other horizons and relabeled "finding a support system."

Four core themes emerged to describe the participants experience of academic social networking. Eliminating redundancies and combining like horizons eliminated 15 horizons, 
leaving a total of 50 horizons. Each horizon was validated against all original participant transcripts. For each horizon, the researcher considered whether it was explicitly expressed in the complete transcription. If not explicitly expressed in all the responses, the invariant constitute was eliminated. For instance, the horizon "creating bonds and friendships" was reported by two participants explicitly, suggested implicitly by two participants, and not recognized explicitly or implicitly by two participants and was therefore eliminated as a horizon of the superordinate theme "acceptance and belonging."

During this validation process one superordinate theme, "feeling of isolation," and all the horizons comprising it were eliminated. Two of the six participants neither explicitly nor implicitly expressed this theme as part of their experience with academic social networking. These final three themes are the central phenomena of this qualitative study: (a) acceptance and belonging; (b) self-validation; (c) drawing from multiple perspectives.

\section{Trustworthiness and Dependability}

Disengagement from the experience is a vital initial process of data collection and analysis and is accomplished through bracketing or epoche (Moustakas, 1994).

Phenomenological research aims to arrive at a common subjective experience, or eidos, of the phenomenon through an analysis of several perspectives. In the study, a common truth of what it means to participate in an academic social network begins with bracketing off prejudgments concerning what this experience entails for the researcher. Knowledge of researcher bias and predisposition allows for a return to the conscious experience as it exists without outside perceptions attached to the meaning.

Trustworthiness is concerned with the data yielding the truth as well as the ability of the researcher to draw accurate conclusions from that data (Golafshani, 2003; Lincoln \& Guba, 1985). Trustworthiness in qualitative research involves examining the truth of a phenomenon by examining a valid sample. Choosing a quality sample to draw data from was accomplished by locating online higher education students active in academic social networking and then by purposefully choosing a sample from that target population utilizing maximum variation.

Rigor was established through a selection of participants appropriate to the research question as well as the maintenance of interview quality (Smith et al., 2009). An interview guide was used to ensure that adequate depth was achieved and the topic was sufficiently covered. A panel of experts in social networking reviewed and provided feedback on the interview guide. The interview guide was also piloted using participants with similar characteristics to the sample population to aid in developing a validated and consistent interview process throughout data collection.

\section{Limitations}

On two separate occasions, 200 students were emailed requesting they volunteer to participate in the study. Ability to enter the academic social network and solicit volunteers to interview opposed to solicitation via email may have broadened the target population and generated greater interest. Several interviewees were unable to meet for the scheduled interview times. Out of the original 11 participants selected only six participated in the interviews.

Research indicates that most themes are present within the first six interviews when participants are purposefully selected: however, the next six transcripts present additional themes totaling $92 \%$ of thematic discovery (Guest, et. al, 2006). Additional interviews may have uncovered themes not represented in the six participants' experiences. Furthermore, the homogeneity of the population may strengthen the validity of the themes found when analyzing 
the six participants' transcripts. All six participants were enrolled in the same university; data regarding experiences in academic social networking across various networks may provide additional insight to this phenomenon.

\section{Results}

The purpose of this qualitative phenomenological study was to explore the lived experiences of online students participating in an academic social network. The specific problem addressed was the lack of knowledge regarding how students use academic social networks in online higher education institutions (Liu et al., 2010). One overarching and exploratory research question was used to guide the study: What meaning does the experience of academic social networking hold for online higher education students? Participants explored their unique reality of the experience of academic social networking via the interview process. Three core themes emerged from data analysis; (1) acceptance and belonging; (2) self-validation; (3) drawing from multiple perspectives. The three core themes are reinforced by the tri-lens theoretical framework: socio-ecological theory (Altback et al., 2005), socio-constructive theory (Hofstetter \& Schneuwly, 2009), and community of inquiry (Arbaugh et al., 2008), and are supported by the review of literature.

\section{Theme 1: Acceptance and Belonging}

Acceptance and belonging involves a feeling of being part of a community where learning occurs through shared and interpersonal communications with peers. Contribution is organic and moves from creator of content to user of content with blurred boundaries between mentor and mentee, resulting in a sense of freedom that comes from choosing one's own role within the community. Acceptance and belonging emerged as a theme describing how students interact in an academic social network. User attitudes regarding the phenomenon suggested a reciprocal relationship between that of the individual user and that of the larger community, consistent with a socio-ecological framework (Granovetter, 1989). Socio-ecological theorists suggest that learning is situated within the context of the relationship between individuals and the community (Greenhow et al., 2009; Ziegler et al., 2013). The students formed communities based on shared interests and the communities in return reinforce the learning through the dialogue which unfolds. Informal and formal learning occur with the student playing the role of both the mentor and the mentee, dependent upon the given topic of discussion.

Communities are not specific groups started by an administrator but communities that form organically out of necessity based on the student population. For this study, the idea of community describes the feeling of acceptance and belonging acquired by both posters and nonposters when engaging in discussion surrounding certain topics of interest in the academic social network. Non-posters describe the academic social networking experience as engaging and feeling like they are part of a group (despite never actually posting any information).

Users seek out other individuals with the same questions, concerns, and characteristics they see reflected in themselves and therefore feel a sense of being in the conversation through the posts of peers with which they feel a connection. One participant explained his role of mentor as he witnessed a peer struggling in a post with a concept with which he was familiar. The student participant never actually replied, however another student in the thread posted the same answer the participant was thinking which provided a level of satisfaction that he knew how to help the peer, even if technically he did not respond to the question. 
Participants reported utilizing the academic social network as a support system when in need of mentoring. Similar behaviors suggested non-posters and posters both benefited from the learning provided through the network, consistent with previous research (Xie, 2013). Nonposters found answers to questions by seeking out peers who shared similar characteristics and had similar questions.

Posters, comprising 17\% of the population in this study, either searched for answers or simply posted questions. Both groups reported utilizing the network in some fashion as a medium for support when struggling either academically or seeking out similar frustrations with degree attainment or questions regarding the career field. The sense of acceptance and belonging to something larger than yourself was strengthened through the support received in the academic social network.

These findings are consistent with previous research suggesting there are implications for non-posting activity increasing student motivation towards learning (Xie, 2013). Wise et al., (2014) and Dennen (2008) recognized the need for research examining non-posting behaviors and how these behaviors interact with student learning and motivation. Findings from this study indicate that both posting and non-posting (or listening) behavior in academic social networks creates a sense of belonging and acceptance with peers that share similar interests.

\section{Theme 2: Self-Validation}

Self-validation is the realization that peers have experienced similar struggles and/or that accomplishments provide motivation toward goal attainment. When students read relatable failures or successes of peers posted to an academic social network it provides a sense of empowerment and aids with degree persistence. The academic social network provides a medium through which a participant can overcome obstacles and persist through difficult times while pursuing their degree. The participants looked for communities of individuals who shared similar career interests or were enrolled in the same degree program. Students reported seeking out peers with whom they could relate to see if they might be encountering similar obstacles toward degree completion. A sense of self-validation emerged when participants believed that their feelings and concerns were shared by others.

Community of Inquiry provided a lens to view social presence within the context of academic social networking. Rogers and Lea (2005) suggested that social presence includes personal identity as well as identity of self within a larger group or community. Findings from this study suggest students that utilize the academic social network as a medium for communication to build a connection with peers with whom they can relate. Previous studies suggested that social presence has a positive relationship with student learning satisfaction; however, insight into how social presence is successfully facilitated in online education remains unclear (Akyol \& Garrison, 2008; Olesova, et al., 2011).

Once self-validation is achieved, self-efficacy - the belief that one can succeed at a given task and an influence on the degree of persistence when confronting obstacles (Bandura, 1997) is the result. When participants saw their own thoughts and feelings reflected in the posts of peers, their sense of self-efficacy was heightened. Participants reported feeling relieved to know that others experienced similar hurdles and expressed motivation upon reading the posts of other students' successes.

The heightened self-efficacy resulting from self-validation, experienced through connections with peers, may influence student retention (Bandura, 1986). Participants connected with peers in the same degree program reported obstacles, including questions about whether 
their degree program was the right fit for them, and doubts about their ability to complete a difficult course. Participants expressed that their peers' posts provided confirmation that they could persist and succeed in their goals. These findings are consistent with the work of Fisher and Baird (2005) which asserted that the integration of web-based learning communities has a "positive influence on student retention in online courses" (p. 88).

Locus of control describes an individual's beliefs about what will ultimately determine goal attainment. A strong internal locus of control coupled with self-efficacy is a strong predictor of student success in online education (Stavredes, 2012). Students with a strong internal locus of control report a belief that success and failure depend upon their own actions. Study participant responses suggest internal locus of control was experienced when describing the self-validation gained through the academic social network (Bandura, 1997).

\section{Theme 3: Drawing from Multiple Perspectives}

Drawing from multiple perspectives involves learning vicariously through the experiences of peers and utilizing various perspectives and resources to formulate new knowledge. Drawing from multiple perspectives emerged as a theme describing the essence of the academic social networking experience. Sixty-seven percent of participants reported using knowledge of others to construct new meanings, consistent with a socio-constructive learning perspective. Socio-constructive learning theorists approach learning as being situated in the social environment of the learner (Hmelo-Silver, et al., 2008). Knowledge is guided by discovery and inquiry in a socio-constructive epistemological view of learning (Phillip, et al., 2007).

Participants in this study reported utilizing the social network as a medium for the knowledge of the group to be shared. Most participants reported an appreciation for the various backgrounds and experiences of peers and used the experiences of others to build their own understanding of concepts. Participants reported searching out information simply out of curiosity, a self-guided learning experience consistent with socio-constructive learning (Phillips et al., 2007).

Participant responses described a socio-constructive learning experience whereas both the relationships formed within the community as well as the environment itself influence knowledge creation. Although $66 \%$ of participants reported not feeling there was an educational benefit from the academic social network, students gained knowledge without viewing this process as necessarily being of educational value. Participants did not always recognize an educational value, possibly because of a perceived association between education and formal process (Jung, 2011). Participants commented on the vast experiences and different backgrounds of peers and perceived a true value in the variation of sources from which information was posted and described. With the vast information available in society, some theorists report a shift in thinking about what constitutes learning (Hodkinson, 2005).

\section{Discussion}

Participant responses to the interviews demonstrate the convergence of theories which provide the theoretical framework of this study: socio-ecological theory, socio-constructive theory, and community of inquiry theory. Interview responses indicated a desire for social connection and knowledge acquisition with peers who share like interests, consistent with the review of literature. Three sub-questions were utilized to draw out the broad data needed to cover the context of the phenomena of the study (Smith et al., 2009). Interpretation of each question 
situated in the theoretical framework and previous research further enhanced the understanding of the essence of the lived experiences of online higher education students utilizing academic social networks.

\section{Sub-question 1. What is the student experience when using an academic social network?}

Participants were asked about their experiences when participating in an academic social network. They reported formulating new concepts based on the varied experiences of peers consistent with the constructive orientation of the community of inquiry model (Akyol et al., 2009). Web 2.0 technologies provide a medium supporting socio- constructive learning theory which places the student at the center of the learning experience (Hofstetter \& Schneuwly, 2009).

Community of inquiry provides a framework for the intersection of three presences which overlap in an online learning environment to create deep and meaningful learning: social presence, teaching presence, and cognitive presence (Garrison et al, 2010). In the CoI framework, teaching presence is defined as "the design, facilitation and direction of cognitive and social processes for the purpose of realizing personally meaningful and educationally worthwhile learning outcomes" (Garrison, Anderson, \& Archer, 2001, p. 5). Eighty-three percent of participant interactions were student-to-student interactions, indicating less of a need for teaching presence in the network as suggested in the CoI framework.

Participants did report taking on a mentoring role, however, with posters describing the creation of new threads to facilitate discussion on topics of interest. Sixty-seven percent of participants, both posters and non-posters, described taking on a mentoring role. Non-posters felt a sense of self-efficacy when engaging in this mentoring process when they witnessed struggling peers receive assistance (even if the study participant was not the one providing assistance).

One participant did not directly mentor another student but felt the reward of being a mentor because of the close feeling of belonging to a group. Therefore, teaching presence defined as "the design, facilitation and direction of cognitive and social processes for the purpose of realizing personally meaningful and educationally worthwhile learning outcomes" (Garrison, Anderson, \& Archer, 2001, p. 5) is implicit within the academic social network and emerges through student-to-student mentoring without the need for a formal faculty presence.

Educationally worthwhile learning outcomes are subjective and witnessed through the existing literature examining the community of inquiry framework in a formal educational context (Akyol et al., 2009; Garrison, Anderson \& Archer, 2001). Participants in this study reported little educational advantage from academic social networking, yet responses indicated gained knowledge based on peers' experiences. S1 described the vast depth of knowledge from peers on the network, "understanding or the breadth of knowledge that is on there ya know, from everyone that is in the community, I think that is the number one greatest experience that I get from the network." S2 explained how she utilized the network as a support system, "when I'm frustrated when I don't know what I am doing and then find the help to get it done it is absolutely liberating."

S3 described the benefits of the network with student-to-student mentoring: "see if anybody has suggestions on how to approach something or um if there has been any um, I will just kind of call it student to student tutoring." S4 explained how peers in the network provide degree program information, "to read other opinions to see what they have read about in the criminal justice field." S4 described how networking provides additional information about her career interest: "had to do with a couple of nursing hot topics and then the other one had to do 
with just frustrations." S5 utilized the network as a support system for degree planning and stated she "read[s] some posts on where other students were discussing should they pursue their masters."

Although participants for this study stated they did not feel a formal educational benefit from academic social networking, $100 \%$ reported utilizing the information in the network informally to assist with educational needs. In an academic social network, participants themselves choose what content is meaningful and develop discussion threads. Coroama's (2011) research indicated a need for flexibility in online education toward an approach incorporating both informal and formal education into the curricula. Study findings suggest the academic social network provides this space for students to informally explore ideas building on previous knowledge, consistent with socio-constructive learning theory, which enhances the overall educational experience.

Sub-question 2. Who or what do students observe in the academic social network?

Participants were asked about who they observe and who they interact with when participating in an academic social network. Due to limited research regarding student activity in academic social networks, previous research regarding populations participating in social networks provided a basis for the literature review. Boyd (2007) defined a social network as a "web-based service[s] that allows individuals to (1) construct a public or semi-public profile within a bounded system, (2) articulate a list of other users with whom they share a connection, and (3) view and traverse their list of connections and those made by others within the system" (p. 211).

Social networks historically provide a virtual location for users of like interests to communicate. What common interests bring users together and how knowledge is disseminated within the network varies based on the site (Boyd, 2007). Boyd (2007) reported that social networks form communities which mirror homogeneous populations according to age, nationality, or education level. This study explored the intrapersonal and interpersonal characteristics of participants, consistent with socio-ecological and community of inquiry frameworks. While the intrapersonal characteristics of participant users illustrate attitudes toward academic social networking, the interpersonal characteristics of users illustrated why they use academic social networking.

Participants did not indicate that age or nationality influenced which peer posts were read or the communities in which posts were shared. The topic of degree program information or career interests determined the communities with which participants connected. Eighty-three percent of participant connections were $100 \%$ virtual with only $17 \%$ reporting physically meeting peers during on-ground courses within the same university prior to participating in the academic social network community. These findings concur with earlier research by Garrison, Anderson, and Archer (2010) which proposed that a shared educational context superseded personal identity and interpersonal relationships when forming connections in online education communities.

Study participants reported communities consisted of peers as opposed to faculty and alumni, who are all provided access to the same academic social network. Seventeen percent of participants reported communicating with faculty while $100 \%$ of participants reported seeking out peers sharing similar career and educational interests. Opposed to seeking knowledge from a person of authority such as faculty or a person of expertise such as alumni, peers sought to learn through individuals with whom they could relate regarding the topic of interest. These findings 
are consistent with socio-constructive learning theory and suggest the learner is at the center of the experience as opposed to an objective learning experience where knowledge is transmitted from one generation to the next (Phillips et al., 2007).

Community of inquiry theory provides a process model suggesting that online learning involves the relationship between social presence, teaching presence, and cognitive presence grounded in a constructivist orientation (Annand, 2011). Social presence is represented though an emotional sense of belonging which develops through progressive stages beginning with identifying with a community, then moving toward purposefully communicating in an environment of trust, and finally developing interpersonal relationships within that community (Garrison et al., 2010).

Consistent with previous research (Annand, 2011), participants in this study described finding acceptance and belonging with peers of like interests, connecting with that community, and developing close bonds with peers within the community. Thirty-three percent of participants reported that a bond or close friendship developed with peers and $100 \%$ of that population comprised of posters. Non-posters reported feeling connected to a community however did not report feelings of close bonds with peers.

Rourke and Kanuka (2009) contended that student engagement in online experiences did not result in "deep and meaningful learning" (p. 24) as previous research by Garrison, Anderson, and Archer (2010) suggested. Annand (2011) posited that learners cannot share common values and goals which are necessary for learning to occur without engaging in group interactions. The study findings indicate that participants' values and goals mirrored those of their peers, and that participating in posting activities was not necessary to feel a sense of engagement. Only when moving toward the last dimension of social presence, forming bonds, were posting activities described.

\section{Sub-question 3. What benefits or drawbacks have students experienced from using an academic social network?}

Participants were asked about why they participate in an academic social network. Consistent with the socio-ecological lens of the study, participants reported utilizing the network as a medium for knowledge construction. Previous research suggested activities vary among social networks (Boyd, 2007). Foster et al.'s (2010) research on virtual communities of practice examined motivations for participation in social networks, finding social connectivity and social enhancement to be the leading motivators for participation in group- based virtual communities. Ardichvili (2008) supported these conclusions, finding motivation factors for participating in communities of practice to include: (1) profession-related benefits such as increased self-esteem and relationship building, and (2) community-based considerations such as shared values and visions.

The study findings identify curiosity about what other students might be doing as a motivator for first becoming active in an academic social network. A review of literature did not present this motivator in previous research on social networking. Participant responses indicated curiosity as a driving factor, with comments such as "just to see what other people are saying," and to "see if something catches my eye." Participants indicated, however, that self-validation was needed to continue visiting the network.

Participants in this study described a sense of "liberation" when finding answers to questions and "wanting to keep the motivation amongst everybody and morale up." That 
participation in the network provided a sense of self-efficacy, a feeling that "if someone else can do it, so can I," was reported across the participants. Participant responses indicated selfvalidation when engaging with students going through struggles similar to their own. Whether or not peers overcame the struggles was not as important to participants as a realization of shared experience. This finding differs from previous reports identifying increased self-esteem as a motivating factor for engaging in communities of practice (Ardichvili, 2008).

Participants did not report any drawbacks to academic social networking and were familiar with navigating the system. Thirty-three percent of participants did recall negative conversations on the network, but said they were not affected by them and continued to search until finding conversations of interest. Thirty-three percent of participants were forty-five or older, and $100 \%$ of that age group indicated they did not understand some of the technical features beyond simple reading and posting activities.

\section{Self-Actualization: The Essence of the Phenomenon}

One common connection found across all individual meanings when layering each of the three themes, which conveys the essence of what it means to participate in an academic social network, is self-actualization. Findings from this study indicate that academic social networks can empower students in distance education by providing a space to engage in social and democratic learning through participation in critical curiosity regarding career and educational goals while enhancing individual growth. When asking what makes this phenomenon unique and why is it important to understand in the context of education, it was discovered that students' view of the world and of their place in the world through participation in the network changed, whether posting or not posting. Acceptance and belonging, self-validation, and multiple perspectives thematically describe the phenomenon of academic social networking as a catalyst to self-actualization.

The findings of this study suggest that the academic social network is organic and in constant flux, driven by individuals who choose to utilize it as part of their educational experience. The resulting communities address various topics that range from career attainment to assignment frustrations to "hot" topics in a career field, and much more. The posts are as broad and differentiated as the participants bring insights from many perspectives based on different experiences, backgrounds, and knowledge.

Students seem to utilize the academic network as a medium for self-growth in an environment where they can control both the area and type of growth desired. Maslow's work, Motivation and Personality (1987), explains a hierarchy of needs individuals must attain before moving forward to the goal of self-actualization. Lower levels of needs include physiological attainment (such as food, water, sleep), safety (security, resources), love and belonging (friendship, family), and esteem (confidence, achievement, respect). According to Maslow, all lower-level needs must be fulfilled before moving to the next level toward the highest level, selfactualization (1987), where an individual's full potential takes place.

Maslow's focus is primarily on the self; however, individuals participating in an academic social network are meeting their needs while also influencing the other participants in the network, forming an ecosystem. The findings from this study suggest that students not only contribute to satisfying their own needs to attain self-actualization but also meet the needs of other members in the community in an organic and circular manner resulting in others' heightened sense of acceptance, belonging, and self-validation. 
Academic social networking sites challenge the epistemological assumptions that constitute knowledge and learning due to their organic and ecological nature. Academic social networking places users in a unique position of both creator and user of content. These sites are a self-organized medium for communication which manifest complex group interactions, demographics and socioeconomic status disappear, and new learning unfolds.

\section{Recommendations}

While enrolment rates continue to rise in online education, persistence toward degree completion is consistently lower than in traditional brick-and-mortar universities. There is a need for education practitioners in online and adult education to share their experiences and lead this paradigm shift toward a collaborative and learner-centered approach to knowledge acquisition in the online environment (Hoskins, 2011). Specifically, the growth in online education warrants a closer examination of how communities form in academic social networks and how influential these communities and the relationships formed in them are regarding student persistence toward degree completion. Peer-to-peer mentoring may be both highly regarded by students and have a distinct effect on retention (Boyle, et al., 2010). Findings from this study support previous research and suggest a socio-constructive and socio-ecological framework for peer mentoring which develops naturally in an academic social network, and which may have implications for student retention, resulting in long-term cost savings for online universities.

\section{Recommendations for Leaders in Education}

Education leaders are encouraged to experiment with social networking outside the purview of marketing, but rather as a tool to foster relationships which support both informal and formal learning outside of the classroom environment. One example may be a mentoring program for incoming freshman who are introduced to peers during the first course of the program. Leaders must be willing to transcend challenges by trying new approaches and utilizing feedback for continual improvement in a fluid organization. Educational leaders need to explore how students are sharing knowledge and educational strategies reflecting best practices within academic social networks in online education.

Traditional universities benefit from a physical connection of the student body to the campus while online universities lack that sense of physical connectedness and social presence. Social integration and community involvement are associated with student persistence and degree completion (Heaney \& Fisher, 2011). Findings suggest that students participating in an academic social network perceive they are accepted and belong to a community of peers whether involved in posting or non-posting behaviors. Education leaders are encouraged to find a virtual space for students to network and create support systems. The costs associated with implementing an academic social network may be offset by an increase in retention rates.

\section{Recommendations for Future Research}

This study provided an understanding of the essence of what it means to participate in an academic social network for online higher education students. Findings from this phenomenological study resulted in recommendations for future research to address unanswered questions. Suggested research will bring a deeper understanding to the findings of this study. Further research is recommended based on the scope and limitations of this qualitative phenomenological study. A gap in literature regarding academic social networking prompted the need for a better understanding of the essence of the experience of online higher education students participating in academic social networks. With the exponential growth in online 
education and subsequently academic social networking the depth of this phenomenon remains unclear.

This study focused on a population in the United States; however, academic social networking is a global phenomenon with institutions utilizing social networking for education and learning in Asia and Europe, among others. Examining the essence of this experience from a global perspective would provide additional context to what it means to participate in an academic social network. Building on this idea, researchers need to examine what learning means in academic social networks and how informal and formal learning interact and unfold in these networks.

Findings were unclear as to whether participants were purposefully seeking out knowledge from various sources reflective of an epistemological belief that knowledge comes from many as opposed to coming from an elite few as is consistent with classical or objectivist learning theory (Tucker \& Courts, 2010). Further research to understand how technological advancements influence epistemological beliefs about what constitutes knowledge is warranted. Cultural and political aspects of human behavior should be examined from a socio-historical theoretical framework to provide an understanding of how knowledge is perceived and what that means for education on a global scale.

Further research is needed to understand non-posting behavior or listening in academic social networks. Specifically, what motivates listeners to participate in academic social networks and what are the differences between the experiences of listeners and posters? Quantitative research would provide insight into how listening behaviors influence student learning and persistence in degree completion. Study findings do not indicate whether students who have a high degree of internal locus of control are more likely to seek validation through the network or whether the network provides a medium for students to feel self-validated resulting in a greater sense of self-efficacy and internal locus of control. Quantitative research is needed to define characteristics of students who participate in the academic social network. More research is needed to fully understand why some students participate in these networks while others do not participate. Additional recommendations based on the study's findings suggest research in the following areas to assist educational leaders and researchers in gaining a deeper knowledge base: (a) social presence and the relationship between social presence and student satisfaction with learning, and (b) the relationship between participation in academic social networks and selfefficacy. Quantitative research is suggested to examine cause and effect relationships to deepen the understanding of the findings from this study.

\section{Conclusion}

Educational leaders are challenged to meet the student demand for online education opportunities while maintaining a rigorous curriculum leading to degree attainment. Academic social networks provide a collaborative medium for communication across time and space. Understanding student experiences of, and attitudes toward, academic social networking can assist educational leaders in realizing the potential of this Web 2.0 tool in the online university. Findings on academic social networking can provide education leaders with guidance when making decisions regarding the use of academic social networks in a university. Educational leaders can assist students toward degree completion by better understanding the student experience and factors that present themselves as challenges in distance education. Academic 
social networks have the capability to assist students with collaboration and build relational communities within the online university.

\section{Declarations}

The author declared no potential conflicts of interest with respect to the research, authorship, and/or publication of this article.

The author received approval from the ethics review board of University of Phoenix for this study.

The author received no financial support for the research, authorship, and/or publication of this article. 


\section{References}

Ardichvili, A. (2008). Learning and knowledge sharing in virtual communities of practice: Motivators, barriers, and enablers. Advances in Developing Human Resources, 10(4), 541-554. https://doi:10.1177/1523422308319536

Akyol, Z. \& Garrison, D. R. (2008). The development of a community of inquiry over time in an online course: Understanding the progression and integration of social, cognitive and teaching presence. Journal of Asynchronous Learning Networks, 12, 3-4.

Ali, A., \& Kohun, F. (2007). Dealing with social isolation to minimize doctoral attrition - a four stage framework. International Journal of Doctoral Studies, 2, 33-29.

http://www.ijds.org/Volume1/IJDSv1p021- 033Ali13.pdf

Annand, D. (2011). Social presence within the community of inquiry framework. International Review of Research in Open and Distance Learning, 12(5), 40-56.

Altbach, P. G., Berdahl, R. O., Gumport, P. J. (2005). American higher education in the twentyfirst century: Social, political, and economic challenges (2nd ed.). John Hopkins University Press.

Arbaugh, J. B., Cleveland-Innes, M., Diaz, S., Garrison, D. R., Ice, P., \& Richardson, J. (2008). Developing a community of inquiry instrument: Testing a measure of the community of inquiry framework using a multi-institutional sample. Internet and Higher Education, 11, 133-136. https://doi.org/10.1016/j.iheduc.2008.06.003

Arnold, N., \& Paulus, T. (2010). Using a social networking site for experiential learning: Appropriating, lurking, modeling and community building. Internet and Higher Education, 13(4), 188-196. https://doi.org/10.1016/j.iheduc.2010.04.002

Bandura, A. (1997). Self-efficacy: The exercise of control. Worth.

Banks, J., Au, K., Ball, A., Bell, P., Gordon, E., Gutierrez, K., Heath, S., et al. (2007). Learning in and out of school in diverse environments (Consensus Report). Learning in Informal and Formal Environment (LIFE) Center. http://life-slc.org/docs/Banks_etal-LIFE-DiversityReport.pdf

Bahk, C., Sheil, A., Rohm, C., \& Lin, F. (2010). Digital media dependency, relational orientation and social networking among college students. (English). Communications of the IIMA, 10(3), 69-78.

Boyle, F., Kwon, J., Ross, C., \& Simpson, O. (2010). Student-student mentoring for retention and engagement in distance education. Open Learning: The Journal of Open, Distance and eLearning, 25(2), 115-130. https://doi.org/10.1080/02680511003787370

Boyd, D. (2011, January 15). The economist debate on social networking [Web log post]. http://www.zephoria.org/thoughts/archives/2008/01/15/the_economist_d.html 
College Board and Art \& Science Group (2009). Social Networking Sites. Student Poll, (7)2, http://professionals.collegeboard.com/data-reports-research/trends/studentpoll/social-networking

Coroama, L. (2011). Reconsidering the relation between the formal and informal learning of the English language in the higher education system. Euromentor, 2(4), 85-91.

Creative Commons Attribution Share-Alike 3.0 (2011). Educational Networking [Weblog post]. http://www.educationalnetworking.com/

Dennen, V. P. (2008). Looking for evidence of learning: assessment and analysis methods for online discourse. Computers in Human Behavior, 24(2), 205-219.

https://doi.org/10.1016/j.chb.2007.01.010

Durak, H. Y. (2020). Cyberloafing in learning environments where online social networking sites are used as learning tools: Antecedents and consequences. Journal of Educational Computing Research, 58(3), 539-569.

Edelmann, N. (2013). Reviewing the definitions of "lurkers" and some implications for online research. Cyberpsychology, Behavior \& Social Networking, 16(9), 645-649.

https://doi.org/10.1089/cyber.2012.0362

EDUCAUSE: Center for Applied Research (2010). The ECAR study of undergraduate students and information technology. Educause. http://www.educause.edu/Resources/ECARStudyfUndergraduateStuden/217333

Eodice, M. \& Gaffin, D. (2008). Let's face Facebook. The National Teaching \& Learning Forum, 17(6), 1-4.

Foster, M. K., Francescucci, A., \& West, B. C. (2010). Why users participate in online social networks. International Journal of E-Business Management, 4(1), 3-19.

Fisher, M. \& Baird, D. E. (2005). Online learning design that fosters student support, selfregulation, and retention. Campus-Wide Information Systems, (22)2, Emerald Group Publishing. https://doi.org/10.1108/10650740610587100

Garrison, D. R., Anderson, T., \& Archer, W. (2010). The first decade of the community of inquiry framework: A retrospective. The Internet and Higher Education, 13(1-2), 5-9. http://www.journals.elsevier.com/the-internet-and- higher-education/

Garrison, D. R., Cleveland-Innes, M., \& Fung, T. S. (2010). Exploring casual relationships between teaching, cognitive and social presence: Student perceptions of community of inquiry framework. Internet and Higher Education, 13, 31-36. https://doi.org/10.1016/j.iheduc.2009.10.002 
Gerow, J. E., Galluch, P. S., \& Thatcher, J. B. (2010). To slack or not to slack: Internet usage in the classroom. Journal of Information Technology Theory and Application, 11(3), 5-24.

Glezou, K., Grigoriadou, M., \& Samarakou, M. (2010). Educational online social networking in Greece: A case study of a Greek educational online social network. The International Journal of Learning, 17(3), 399-420. http://ijl.cgpublisher.com/product/pub.30/prod.2668

Greenhow, C., Robelia, B., \& Hughes, J. E. (2009). Learning, teaching, and scholarship in a digital age. Educational Researcher, 38(246-258). https://doi.org/10.3102/0013189X09336671

Guest G., Bunce A., Johnson L. (2006). How many interviews are enough? an experiment with data saturation and variability. Field Methods. (18) pp. 59-82.

Heaney, A., \& Fisher, R. (2011). Supporting conditionally admitted students: A case study of assessing persistence in a learning community. Journal of the Scholarship of Teaching \& Learning, 11(1), 62-78. http://josotl.indiana.edu/issue/view/166

Heo, G. \& Lee, R. (2013). Blogs and social network sites as activity systems: Exploring adult informal learning process through activity theory framework. Journal of Educational Technology \& Society, 16(4), 133-145.

Hmelo-Silver, C. E., Chernobilsky, E., \& Jordan, R. (2008). Understanding collaborative learning processes in new learning environments. Instructional Science, 36(5-6), https://doi.org/10.1007/s11251-008-9063-8

Hodkinson, P. (2005). Learning as cultural and relational: moving past some troubling dualisms. Cambridge Journal of Education, 35(1), 107-119. https://doi.org/10.1080/0305764042000332524

Holland, A. A. (2019). Effective principles of informal online learning design: A theory-building metasynthesis of qualitative research. Computers \& Education, 128, 214-226. https://doi.org/10.1016/j.compedu.2018.09.026

Hofstetter, R., \& Schneuwly, B. (2009). Knowledge for teaching and knowledge to teach: two contrasting figures of new education: Claparede and Vygotsky. Paedagogica Historica, 45, 605629. https://doi.org/10.1080/00309230903100973

Hoskins, B. (2011). Demand, growth, and evolution. Journal of Continuing Higher Education, 59(1), 57-60.

Ilkyu, H., \& Chonggun, K. (2014). Understanding user behaviors in social networking service for mobile learning: A case study with twitter. Malaysian Journal of Computer Science, 27(2), 112-123. 
Ivankova, N. V., \& Stick, S. L. (2007). Students' persistence in a distributed doctoral program in educational leadership in higher education: A mixed methods study. Research in Higher Education, 48(1), 93-135. https://doi.org/10.1007/s11162-006- 9025-4

Jones, S. (2002). The internet goes to college. Pew Internet \& American Life. USDLA Journal, 16(10).

Jung, Y. (2011). Education as a ubiquitous learning web, immersed in living. Journal of Unschooling \& Alternative Learning, 5(9), 38-56.

Kabilan, M. K., Ahamad, N., \& Abidin, M. J. (2010). Facebook: An online environment for learning of English in institutions of higher education? Internet and Higher Education, 13, 179187. https://doi.org/10.1016/j.iheduc.2010.07.003

Kaya, T. (2010). New College Social Networks, Unlike Facebook, Foster Academic Interaction. The Chronicle of Higher Education. http://chronicle.com/article/New-College-NetworksUnlike/124871/

Ke, F. (2010). Examining online teaching, cognitive, and social presence for adult students. Computers \& Education. 55(4), 808-820. https://doi.org/10.1016/j.compedu.2010.03.013

Livingstone, D. W. (2001). Adults' informal learning: Definition, findings, gaps and future research. Canada: Human Resources Development. Center for the Study of Education and Work. https://tspace.library.utoronto.ca/retrieve/4484

Liu, M., Kalk, D., Kinney, L., \& Orr, G. (2010, October). How Web 2.0 technologies are used in higher education: An updated review of literature. In E-Learn: World Conference on E-Learning in Corporate, Government, Healthcare, and Higher Education (pp. 2604-2615). Association for the Advancement of Computing in Education (AACE).

Lowenthal, P. R. (in press). Social presence. In P. Rogers, G. Berg, J. Boettcher, C. Howard, L. Justice, \& K. Schenk (Eds.), Encyclopedia of distance and online learning (2nd ed.). Information Science Reference. http://www.patricklowenthal.com/publications/socialpresenceEDOLpreprint.pdf

Marshall, C., \& Rossman, G. B. (2006). Designing qualitative research (4th ed.) Sage Publications.

Maslow, A. H. (1987). Motivation and personality (3rd. ed.). Harper Collins Publishers.

Merriam, S. B. (2009). Qualitative research: A guide to design and implementation. Revised and expanded from qualitative research and case study applications in education. John Wiley \& Sons, Inc. 
Morrison, M., \& McMillan, S. (2010). Oh, user, who art thou: An examination of behaviors and characteristics of consumers in the context of user generated content. American Academy of Advertising Conference Proceedings, 77.

Morrison, M., Hyuk Jun, C., \& McMillan, S. J. (2011). Shading in the gray: Profiling media use patterns of posters, lurkers, and networkers. American Academy of Advertising Conference Proceedings, 56-58.

Moustakas, C. (1994). Phenomenological research methods. Thousand Oaks, CA: Sage Publications.

Muller, M. J., Freyne, J., Dugan, C., Millen, D. R., \& Thom-Santelli, J. (2009). Return On Contribution (ROC): A metric for enterprise social software. In ECSCW 2009 (pp. 143-150). Springer.

National Center for Educational Statistics (2006). Adult education survey of the 2005 National Household Education Survey Program. http://nces.ed.gov/pubs2006/2006077.pdf

Neuman, W. (2011). Social research methods: Qualitative and quantitative approaches (7th ed.). Allyn \& Bacon.

Olesova, L. A., Weasenforth, D., Richardson, J. C., \& Meloni, C. (2011). Using asynchronous instructional audio feedback in online environments: A mixed methods study. Journal of Online Learning and Teaching. 7(1). http://jolt.merlot.org/vol7no1/olesova 0311.htm

O’Neill, T. A., Hambley, L. A., \& Chatellier, G. S. (2014). Cyberslacking, engagement and personality in distributed work environments. Computers in Human Behavior, 40,152-160.

Oregon, E., McCoy, L., \& Carmon-Johnson, L. (2018). Case analysis: Exploring the application of using rich media technologies and social presence to decrease attrition in an online graduate program. Journal of Educators Online, 15(2), 103-115. https://doi.org/10.9743/jeo.2018.15.2.7

Phillips, P. Wells, J., Ice. P., Curtis, R., \& Kennedy, R. (2007). A case study of the relationship between socio-epistemological teaching orientations and instructor perceptions of pedagogy in online environments. Electronic Journal for the Integration of Technology in Education, (6) 327.

Radovan, M. (2019). Should I stay, or should I go? Revisiting student retention models in distance education. Turkish Online Journal of Distance Education, 20(3), 29-40.

Rajagopal, K., Brinke, D. J., \& Sloep, P. B., (2012). Understanding personal learning networks: Their structure, content and the networking skills needed to optimally use them. First Monday. (17)1. http://www.uic.edu/htbin/cgiwrap/bin/ojs/index.php/fm/article/viewArticle/3559/3131 
Rogers, P., Berg, G., Boettcher, J. Howard, C., Justice, L., \& Schenk, K. (2009), Encyclopedia of distance and online learning (2nd ed.). Information Science Reference. Hershey, NY.

Rogers, P. \& Lea, M. (2005). Social presence in distributed group environments: The role of social identity. Behavior \& Information Technology, 24(2): 151-158. https://doi.org/10.1080/01449290410001723472

Rubenson, K. \& Desjardins, R. (2009). The impact of welfare states' regimes on barriers to participation in adult education. Adult Education Quarterly, 59(3), 187-207.

Rourke, L., \& Kanuka, H. (2009). Learning in communities of inquiry: A review of the literature. Journal of Distance Education, 23(1), 19-48.

Saykili, A. (2019). Higher education in the digital age: The impact of digital connective technologies. Journal of Educational Technology and Online Learning, 2(1), 1-15.

Scarlett-Ferguson, H. (2011). Leadership in Distance Education: Do We Need a Leadershift? In Proceedings of World Conference on E-Learning in Corporate, Government, Healthcare, and Higher Education 2011 (pp. 2249-2253). AACE.

Smith J. A., Flowers, P., \& Larkin, M. (2009). Interpretative phenomenological analysis: Theory, method and research. Sage Publications.

Smith, S., Salaway, G., Caruso, J., \& Katz, R. N. (2009). The ECAR study of undergraduate students and information technology. EDUCAUSE Center for Applied Research, (6). http://www.educause.edu/ecar

Smith, M. C. \& Smith, T. J. (2008). Low-education adults' participation in informal activities: Relationships with selected demographic characteristics. Adult Basic Education and Literacy Journal, 2(2), 67-73.

Short, J., Williams, E., \& Christie, B. (1976). The social psychology of telecommunications. John Wiley \& Sons.

Stavredes, T. (2014). Effective online teaching, foundations and strategies for student success. John Wiley \& Sons, Inc.

Tanriverdi, O. \& Daraca, F. (2018). Investigating the relationships between adolescents' levels of cognitive absorption and cyberloafing activities according to demographic characteristics. The Turkish Journal on Addictions. 5(2), 285-315.

The E-Listening Project (2011). E-Listening: Research Project. https://www.sfu.ca/ afw3/research/e-listening/index.html 
Valdez, G. F. D., Cayaban, A. R. R., Al-Fayyadh, S., Korkmaz, M., Obeid, S., Sanchez, C. L. A., Ajzoon, M. B., Fouly, H., \& Cruz, J. P. (2020). The utilization of social networking sites, their perceived benefits and their potential for improving the study habits of nursing students in five countries. BMC Nursing, 19(1), 1-14. https://doi.org/10.1186/s12912-020-00447-5

Walther, J. B. (1992). Interpersonal effects in computer-mediated interaction: A relational perspective. Communication Research, 19, 52-90. https://doi.org/10.1177/0093650212438097

Wise, A.F., Hausknecht, S.N. \& Zhao, Y. (2014). Attending to others' posts in asynchronous discussions: Learners' online "listening" and its relationship to speaking. International Journal of Computer Supported Collaborative Learning. (9), 185-209. https://doi.org/10.1007/s11412014-9192-9

Xie, K. (2013). What do the numbers say? The influence of motivation and peer feedback on students' behaviour in online discussions. British Journal of Educational Technology, 44(2), 288301. https://doi.org/10.1111/j.1467-8535.2012.01291 


\section{Appendix A Academic Social Networking}

I am looking for participants who are actively involved in an academic social network, whether that means posting, reading others posts, starting communities, or whatever activities you may take part within the network. I will be choosing a small sample to interview based on responses received in this survey. Thank you for participating in my short survey. An academic social network is social network within online university. Only students attending the university, faculty, and possibly alumni have access to this network. These social networks are available for students to collaborate and communicate with each other as well as faculty and alumni. Participation in an academic social network includes observing others posts and interactions as well as posting and sharing information.

Which best describes the amount of time you spend actively involved in the university's academic social network?

- I participate in the academic social network less than one time per week.

- I participate in the academic social network to see what is new at least once a day.

- I participate in the academic social network several times throughout the day.

Describe in greater detail what you typically do in the academic social network. Do you prefer to read others posts and see what is going on or do you post and interact with other users? You may do a combination of both, or another activity not described here.

What degree program are you currently enrolled in?

What year are you in your degree program?

$1^{\text {st }}$

$2^{\text {nd }}$

$3^{\text {rd }}$

$4^{\text {th }}$

Other

Which of the following age ranges do you fall under?

$18-24$

25-30

31-34

$35-40$

$41-45$

$46+$

What is your gender?

Male

Female 